Revista de MATEMÁticA: TeORÍA y APliCACIONEs 2017 24(2) : 277-286

CIMPA - UCR ISSN: 1409-2433 (PRINT), 2215-3373 (ONLINE)

\title{
MODELO ESTOCÁSTICO PARA LA EPIDEMIA DEL VIH/SIDA
}

\section{STOCHASTIC MODEL FOR VIH/AIDS EPIDEMICS}

\author{
ERICK MANUEl Delgado-MoyA* \\ AYMÉE MARRERO-SEVERO ${ }^{\dagger}$
}

Received: 3/Aug/2016; Revised: 28/Apr/2017;

Accepted: 28/Apr/2017

Revista de Matemática: Teoría y Aplicaciones is licensed under a Creative Commons Reconocimiento-NoComercial-Compartirigual 4.0 International License.

Creado a partir de la obra en http://www.revistas. ucr.ac.cr/index.php/matematica

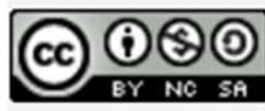

${ }^{*}$ Facultad de Matemática y Computación, Universidad de La Habana. La Habana, Cuba. EMail: erickdelgadomoya@gmail.com

${ }^{\dagger}$ Misma dirección que/Same address as: E-Mail: aymee@ matcom.uh.cu 


\title{
Resumen
}

La epidemia del VIH/SIDA le ha costado a la humanidad innumerables pérdidas tanto materiales como humanas, su estudio se ha convertido en una prioridad para la comunidad científica mundial, con la idea de establecer una política efectiva de control y posteriormente de erradicación. En este trabajo se propone un modelo con Cadenas de Markov basado en el análisis de la matriz de transición con vista a diseñar una estrategia futura para reducir la aparición de nuevos casos y disminuir la letalidad de esta epidemia.

Palabras clave: matriz de transición; VIH; SIDA; estrategia de control.

\begin{abstract}
The HIV/AIDS has cost humanity countless material and human losses. Study its dynamics has become a priority for the global scientific community, with the idea of establishing an effective control policy and subsequently analyzing its eradication. This paper presents a model based on transition matrix of Markov chains with the objective to design a future strategy to reduce the appearance of new cases and decrease the lethality of this epidemic.
\end{abstract}

Keywords: transition matrix; HIV; AIDS; control strategy.

Mathematics Subject Classification: 60G07, 60G25, 62M05, 92B05, 92D30.

\section{Introducción}

El estudio y modelado matemático de las enfermedades infecciosas es un problema que ha sido abordado frecuentemente a lo largo de la historia para ayudar a plantear estrategias en aras de mejorar la prevención, detección temprana y tratamiento de los afectados, así como de establecer medidas de control de su evolución, [2], [5], [7], porque representan uno de los problemas de mayor transcendencia que atañe a las autoridades sanitarias.

En este contexto, existen varios trabajos en la literatura que analizan la epidemia de VIH/SIDA, que es, desde todo punto de vista, un hecho sin precedentes en la historia de la humanidad, [13], [6]. Su rápida diseminación por el mundo, las bajas que ha causado a su paso y los riesgos que implica para la calidad de vida humana la sitúan entre las enfermedades más temibles en la actualidad. Ha movilizado más recursos e investigaciones, que cualquier otra epidemia, por la ausencia de cura, la rapidez de su avance, las diversas formas de contagio que dependen principalmente de la conducta sexual de los individuos y los cambios degenerativos que afectan la salud del enfermo [9], [12]. 
Por el gran impacto social que genera la gravedad y magnitud de esta pandemia, desde su aparición, ha sido motivo de preocupación para la comunidad científica mundial, del que no escapa el interés por su modelación y análisis matemático, en aras de aportar esencialmente a las políticas de control de su desarrollo. En la literatura se han propuesto distintos modelos de la epidemia en función de los factores que se pueden tener en cuenta o las técnicas utilizadas ([1], [13], [3], [4], [6], entre muchos otros).

La mayoría de las investigaciones sobre el VIH/SIDA, se dedicaron al entendimiento de su ocurrencia y presentación en las poblaciones y sus entornos. Posteriormente y hasta la actualidad las investigaciones se han concentrado en la exploración de su transmisión del VIH en grupos particulares que han facilitado la proliferación del virus, y el impacto económico en los sistemas de salud y el mercado laboral [9], [12].

El estudio del VIH/SIDA en el caso de Cuba es especialmente interesante ya que la enfermedad presenta una incidencia muy baja, especialmente si se compara con el resto de los países del Caribe, segunda zona de mayor proliferación mundial, [6], [4]. Según el Anuario Estadístico de Salud de Cuba [10], en el 2014 más del 90\% de los casos detectados de VIH/SIDA fueron en estadios precoces, el SIDA se muestra como la vigésima causa de muerte en Cuba en general, siendo la decimosexta causa de muerte en el sexo masculino y la vigésimoctava en el sexo femenino. En ese mismo año hubo 344 muertes a causa del SIDA, de ellas, 290 hombres y 54 mujeres.

En este trabajo se presentan preliminares sobre Cadenas de Markov, empleadas para el análisis de la matriz de transición en un modelo de transmisión del VIH/SIDA. Además se proponen estrategias de control y las conclusiones obtenidas. Las experimentaciones computacionales se realizaron con el software Matlab 2013a.

\section{Preliminares}

Las cadenas de Markov se deben al matemático ruso A.A. Markov alrededor de 1905. Su intención era crear un modelo probabilístico para analizar las vocales en poemas y textos literarios. El éxito del modelo propuesto por Markov radica en que es lo suficientemente complejo como para describir ciertas características no triviales de algunos sistemas pero al mismo tiempo sencillo para ser analizado matemáticamente [16], [14].

Definición 1 Una cadena de Markov es un proceso estocástico a tiempo discreto $\left\{x_{n}: n=0,1,2, \ldots\right\}$ con espacio de estado discreto, y que satisface 
la propiedad de Markov, esto es, para cualquier $n \geq 0$, y para cualesquiera estados $x_{0}, x_{1}, \ldots, x_{n+1}$, se cumple

$$
P\left(x_{n+1} \mid x_{0}, x_{1}, \ldots, x_{n}\right)=P\left(x_{n+1} \mid x_{n}\right) .
$$

Esto quiere decir que el proceso al tiempo futuro $n+1$ depende únicamente del estado de proceso al tiempo $n$, y no depende de los estados en los tiempos pasados $0,1,2, \ldots, n-1$.

En una cadena definida con $m$ posibles estados $E_{1}, E_{2}, \ldots, E_{m}$, se puede introducir la notación:

$$
p_{i j}=P\left(x_{n}=j \mid x_{n-1}=i\right), \quad i, j=1, \ldots, m,
$$

para definir la probabilidad condicional de que estando en el estado $i$ en el momento anterior inmediato pase al estado $j$.

- Si $p_{i j}>0$ entonces el estado $E_{i}$ está comunicado con el $E_{j}$. Si, además, $p_{j i}>0$ entonces la comunicación es mutua.

Los $p_{i j}$ se denominan probabilidades de transición y todas ellas forman la matriz de transición de dimensión $m \times m$ :

$$
T=\left[p_{i j}\right]
$$

Esta matriz cumple las siguientes propiedades:

- Cada fila de la matriz es una distribución de probabilidad

$$
\sum_{j=1}^{m} p_{i j}=1 .
$$

- La potencia de la matriz de transición se puede tomar como:

$$
T^{n}=C L^{n} C^{-1},
$$

$L$ : matriz diagonal con los valores propios de $\mathrm{T}$.

$C$ : matriz que contiene los vectores propios asociados a los valores propios correspondientes.

Las aplicaciones en la investigación biomédica de las cadenas de Markov han sido múltiples, tanto en la experimentación animal [17], como en los estudios humanos ([15], [8]). 


\section{Modelo general}

Para el estudio de la epidemia, definimos los cuatro posibles estados por lo que transitará un individuo, $S$ : susceptible, $I$ : infectado, $N$ : muerte natural, y $E$ : muerte por la enfermedad. Establecemos una cadena de Markov con los estados anteriores, considerando años, la unidad de tiempo $n$.

Denotemos:

- $\alpha$ : probablidad de mantenerse en el estado de susceptible.

- $\beta$ : probabilidad de mantenerse en el estado de infectado.

- $\mu$ : probabilidad de muerte natural.

- $\gamma=P\left(x_{n}=I \mid x_{n-1}=S\right)$ : probabilidad de estando en el estado susceptible pasar al estado de infectado.

- $\epsilon=P\left(x_{n}=E \mid x_{n-1}=I\right)$ : probabilidad de muerte por la enfermedad dado que está en el estado infectado.

\section{Observaciones:}

1. Se tiene $\alpha+\gamma+\mu=1, \beta+\mu+\epsilon=1$ para que se cumplan las propiedades de la matriz de transición.

2. La muerte natural tendrá la misma probabilidad condicional si partimos del estado de susceptible o infectado.

Las relaciones existentes entre los posibles estados dadas por las probabilidades de tránsito, se representa en la siguiente tabla:

\begin{tabular}{c|cccc}
\hline \hline Estados & $S$ & $I$ & $N$ & $E$ \\
\hline$S$ & $\alpha$ & $\gamma$ & $\mu$ & 0 \\
$I$ & 0 & $\beta$ & $\mu$ & $\epsilon$ \\
$N$ & 0 & 0 & 1 & 0 \\
$E$ & 0 & 0 & 0 & 1 \\
\hline \hline
\end{tabular}

La matriz de transición $T$ de este proceso tiene la forma:

$$
\left(\begin{array}{llll}
\alpha & \gamma & \mu & 0 \\
0 & \beta & \mu & \epsilon \\
0 & 0 & 1 & 0 \\
0 & 0 & 0 & 1
\end{array}\right)
$$


A continuación se presenta una comparación entre los valores de las probabilidades de transición del estado susceptible al infectado y del estado muerte por enfermedad a partir del estado infectado al paso del tiempo. Los parámetros fueron tomados fijos ( $\alpha=0.25, \gamma=0.65, \mu=0.1, \beta=0.7$, y $\epsilon=0.2$ ), de forma hipotética con el objetivo de construir un escenario factible para el desarrollo de la epidemia. Se tomó el valor de la probabilidad $\alpha$ de mantenerse en el estado susceptible, menor que la probabilidad $\gamma$ de infectarse siendo susceptible.

\begin{tabular}{l|ll}
\hline \hline Tiempo & \multicolumn{1}{|c}{$\gamma$} & \multicolumn{1}{c}{$\epsilon$} \\
\hline$n=10$ & 0.00227840690 & 0.79921875 \\
$n=20$ & 0.000002225240047 & 0.799999237050547 \\
$n=30$ & 0.000000002173086 & 0.799999999254942 \\
$n=40$ & 0.000000000002122 & 0.799999999999272 \\
\hline \hline
\end{tabular}

Tabla 1: Variación de $\gamma$ y $\epsilon$ en el tiempo $(n)$.

La probabilidad $\gamma$, a pesar de que va disminuyendo con el paso del tiempo, aún ocupa un lugar significativo en la dinámica de la epidemia y en la aparición de nuevos casos, provocando que crezca la probabilidad de muerte por la enfermedad. Estos resultados avalan la necesidad de establecer alguna estrategia de control sobre dichas probabilidades, ver Tabla 1.

\section{Estrategias de control}

\subsection{Control con tratamiento}

La estrategia de control se establece sobre la cantidad de muertes por la enfermedad al paso del tiempo, o sea, se aplica tratamiento a los pacientes con el objetivo de alargar su vida, pero manteniendo la calidad de la misma. Para ello en el mundo entero se aplica la terapia antirretroviral de gran actividad (TARGA), que no es más que el uso de varios fármacos con el objetivo de controlar la expansión del virus en el organismo. Los criterios para la aplicación de terapia pueden ser diversos dependiendo del sistema de salud de cada país o región, entre ellos pueden destacarse [2]:

- Conteo de células CD4 entre 150 y 350 por milímetro cúbico de sangre.

- Personas infectadas a la vez por VIH y por el virus de la hepatitis B o Virus de la hepatitis C.

- Personas con parejas que son VIH negativas, personas con más de 50 años, etc. 
La idea de esta estrategia es la ponderación del parámetro $\epsilon$, que tributa a la eficacia con la que se va aplicar el control (tratamiento). La matriz de transición para la aplicación de control con coeficiente de ponderación $s$ es:

$$
T_{\epsilon}=\left(\begin{array}{cccc}
\alpha & \gamma & \mu & 0 \\
0 & \beta & \mu & (1-s) \epsilon \\
0 & 0 & 1 & 0 \\
0 & 0 & 0 & 1
\end{array}\right)
$$

Haciendo variar el tiempo $n$ y trabajando con distintos tipos de eficacias de control $s$, se obtuvieron los resultados mostrados en las Tablas 2 y 3.

\begin{tabular}{l|ccc}
\hline \hline Tiempo & $s=0.1$ & $s=0.2$ & $s=0.3$ \\
\hline$n=10$ & 0.780958702023144 & 0.758622444137909 & 0.730657794146233 \\
$n=20$ & 0.782605216929038 & 0.761890621541738 & 0.736790200379170 \\
$n=30$ & 0.782608688317894 & 0.761904700987483 & 0.736841669625796 \\
$n=40$ & 0.782608695636711 & 0.761904761642328 & 0.736842101606812 \\
\hline \hline
\end{tabular}

Tabla 2: Variación de $\epsilon$ en el tiempo $(n)$ con estrategia de control.

\begin{tabular}{l|ccc}
\hline \hline Tiempo & $s=0.1$ & $s=0.2$ & $s=0.3$ \\
\hline$n=10$ & 0.00434058690748 & 0.007935678337621 & 0.013988152097806 \\
$n=20$ & 0.000009151542868 & 0.000018559226468 & 0.000117403904240 \\
$n=30$ & 0.000000009371580 & 0.000000147283552 & 0.000000985370224 \\
$n=40$ & 0.000000000040679 & 0.00000000634504 & 0.000000008270206 \\
\hline \hline
\end{tabular}

Tabla 3: Variación $\gamma$ en el tiempo $(n)$ con estrategia de control.

Considerando un período de 40 años, se obtuvo reducción en la probabilidad de muerte por enfermedad y con mejor eficacia es más aceptable, lo que cumple con el objetivo deseado. Sin embargo, la probabilidad de pasar del estado susceptible a infectado aumenta en comparación al modelo sin control, este comportamiento puede atribuirse a que como se reduce la muerte por enfermedad, aumenta el número de infectados interactuando con los susceptibles, lo que también produce que disminuya la probabilidad de mantenerse en el estado susceptible. A nivel experimental, con esta estrategia de control se cumplen los objetivos esperados, sin embargo, al aumentar la probabilidad de infectación, la epidemia seguirá propagándose. 


\subsection{Control combinado}

La idea está en combinar dos estrategias de control, la presentada anteriormente en la sección [4.1] y la de establecer control sobre los infectados. Para controlar el contagio por vía sexual, que es el principal modo de adquisición de la enfermedad, se definen dos métodos fundamentales [4]:

1. Métodos Naturales: La abstinencia o no práctica de sexo, esta puede ser periódica o la pareja se abstiene de tener sexo en los días fértiles, que es la única manera $100 \%$ segura de prevenir el embarazo y las ITS.

2. Métodos no Naturales: Uso de preservativos, que se pueden utilizar en el sexo vaginal, anal y oral.

Para esta estrategia de control se propone la siguiente matriz de transición:

$$
T_{\epsilon, \gamma}=\left(\begin{array}{cccc}
\alpha & \left(1-s_{1}\right) \gamma & \mu & 0 \\
0 & \beta & \mu & (1-s) \epsilon \\
0 & 0 & 1 & 0 \\
0 & 0 & 0 & 1
\end{array}\right)
$$

Los resultados, a nivel experimental, muestran que la estrategia combinada es más eficiente pues logra reducir tanto las muertes por la enfermedad como el número de infectados, lo que debe permitir una desaceleración de la epidemia. En la Tabla 4 se muestra los resultados al aplicar el control combinado para $s_{1}=0.6$ y $s=0.3$ y se compara con el estudio sin control en cuanto a la probabilidad de transmisión.

\begin{tabular}{l|ll}
\hline \hline Tiempo & $s_{1}=0.6, s=0.3$ & Sin control \\
\hline$n=10$ & 0.001953124800000 & 0.00227840690 \\
$n=20$ & 0.000001907348633 & 0.000002225240047 \\
$n=30$ & 0.000000001862645 & 0.000000002173086 \\
\hline \hline
\end{tabular}

Tabla 4: Variación de $\gamma$ con estrategia de control combinada.

Lo obtenido en nuestras experimentaciones, muestra que aplicando ambos controles de manera simultánea se logra reducir las muertes por la epidemia y además reducir el número de casos que pasarán al estado de infectado, lo que significaría, controlar la epidemia. 


\section{Conclusiones}

En este artículo se presentan modelos matemáticos basados en la matriz de transición de una cadena de Markov para la dinámica de tratamiento y de contagio del VIH/SIDA. Esta herramienta matemática permite modelar y simular el comportamiento de la epidemia, gracias al análisis en el tiempo de los principales parámetros que caracterizan la dinámica de transmisión. Esta propuesta es aplicable a otras epidemias, teniendo en cuenta la correcta selección de las probabilidades y tasas que se estudian, así como a otras regiones, países y sociedades, para tributar a una política de control eficiente.

Los resultados obtenidos y las comparaciones presentadas muestran las diferencias en la eficacia de las estrategias de control aplicadas sobre los parámetros de mayor influencia en la transmisión.

Se recomienda extender el estudio a otras epidemias.

\section{Referencias}

[1] Arazoza, H. de; Lounes, R.; Pérez, J.; Hoang, T. (2003) "What percentage of the Cuban HIV-AIDS epidemic is known?", Revista Cubana de Medicina Tropical 55(1): 30-37.

[2] Bailey, N.T. (1975) The Mathematical Theory of Infectious Diseases and its Applications. Ch. Griffin and Company Ltd., London.

[3] Barrios, J.; Arazoza, H. de; Marrero, A.; Baguer, M.L. (2010) "Parameters estimation in HIV/AIDS epidemiological models", Revista de Matemática: Teoría y Aplicaciones 17(2) : 143-158.

[4] Delgado, E.M.; Marrero, A. (2016) "Modelos matemáticos de VIH/SIDA definidos por EDOs y con estrategia de control", sometido a Revista Ciencias Matemáticas.

[5] Diekmann, O.; Heesterbeek, J.A.P. (2000) Mathematical Epidemiology of Infectious Diseases: Model Building, Analysis and Interpretation. Wiley, New York.

[6] Garaluz, E.G. (2014) Modelado Matemático de Sistemas Dinámicos en Epidemiología, Tesis Docotral, Universidad de Málaga, Málaga, España.

[7] Hsieh, Y.-H.; Arazoza, H. de; Lounes, R.; Joanes, J. (2005) "A class of methods for HIV contact tracing in Cuba: Implications for intervention and 
treatment", in: W.-Y. Tan \& W. Hulin (Eds.) "Deterministic and Stochastic Models for AIDS Epidemics and HIV Infection with Interventions", Word Scientific, Singapore: 1-16.

[8] Kopycka-Kȩdzierawski, D.T.; Billings R.J. (2006) "Application of nonhomogeneous Markov models for analysing longitudinal caries risk", Community Dentistry and Oral Epidemiology 34(2): 123-129.

[9] Lamptey, P.; Johnson, J.L.; Khan, M. (2006) "El desafío mundial del VIH y el SIDA", Population Bulletin 61(1): 1-28.

[10] Ministerio de Salud Pública. Anuario Estadístico de Salud. República de Cuba, versiones de 2010, 2011, 2012, 2013, 2014

[11] Murray, J. (2002) Mathematical Biology, 3rd ed. Springer, New York.

[12] Shiffman, J. (2006) "Has donor prioritization of HIV/AIDS control displaced aid for other health causes?", Public Health and Humna Rights, PAHA 134th Annual Meeting and Exposition, November 4-8, Boston MA.

[13] Tan, W.-Y.; Wu, H. (2005) Deterministic and Stochastic Models of AIDS Epidemics and HIV Infections with Intervention. World Scientific, Singapore.

[14] Taylor, H.M.; Karlin, S. (1998) An Introduction to Stochastic Modeling. Academic Press, San Diego CA.

[15] Trajstman, A.C. (2002) "A Markov chain model for Newcastle disease and its relevance to the intracerebral pathogenicity index", Biometrical Journal 44(1): 43-57.

[16] Tudor, C. (1994) Procesos Estocásticos. Aportaciones Matemáticas, Serie Texto 2, Sociedad Matemática Mexicana, México D.F.

[17] Yang H.C.; Chao, A. (2005) "Modelling animal's behavioural response by Markov chain models for capture-recapture experiments", Biometrics 61: 1010-1017. 